\title{
Multimodal processing of emotional information in 9- month-old infants II: prenatal exposure to maternal anxiety
}

Citation for published version (APA):

Otte, R. A., Donkers, F. C. L., Braeken, M. A. K. A., \& Van den Bergh, B. R. H. (2015). Multimodal processing of emotional information in 9-month-old infants II: prenatal exposure to maternal anxiety. Brain and Cognition, 95, 107-117. https://doi.org/10.1016/j.bandc.2014.12.001

Document status and date:

Published: 01/01/2015

DOI:

10.1016/j.bandc.2014.12.001

Document Version:

Publisher's PDF, also known as Version of record

\section{Document license:}

Taverne

Please check the document version of this publication:

- A submitted manuscript is the version of the article upon submission and before peer-review. There can be important differences between the submitted version and the official published version of record.

People interested in the research are advised to contact the author for the final version of the publication, or visit the DOI to the publisher's website.

- The final author version and the galley proof are versions of the publication after peer review.

- The final published version features the final layout of the paper including the volume, issue and page numbers.

Link to publication

\footnotetext{
General rights rights.

- You may freely distribute the URL identifying the publication in the public portal. please follow below link for the End User Agreement:

www.umlib.nl/taverne-license

Take down policy

If you believe that this document breaches copyright please contact us at:

repository@maastrichtuniversity.nl

providing details and we will investigate your claim.
}

Copyright and moral rights for the publications made accessible in the public portal are retained by the authors and/or other copyright owners and it is a condition of accessing publications that users recognise and abide by the legal requirements associated with these

- Users may download and print one copy of any publication from the public portal for the purpose of private study or research.

- You may not further distribute the material or use it for any profit-making activity or commercial gain

If the publication is distributed under the terms of Article $25 \mathrm{fa}$ of the Dutch Copyright Act, indicated by the "Taverne" license above, 


\title{
Multimodal processing of emotional information in 9-month-old infants II: Prenatal exposure to maternal anxiety
}

\author{
R.A. Otte ${ }^{a}$, F.C.L. Donkers ${ }^{\mathrm{a}, \mathrm{b}, \mathrm{c}}$, M.A.K.A. Braeken ${ }^{\mathrm{a}, \mathrm{d}}$, B.R.H. Van den Bergh ${ }^{\mathrm{a}, \mathrm{e}, \mathrm{f}, *}$ \\ ${ }^{a}$ Department of Psychology, Tilburg University, PO Box 90153, 5000 LE Tilburg, The Netherlands \\ ${ }^{\mathrm{b}}$ Department of Psychiatry, University of North Carolina at Chapel Hill, 101 Manning Drive, Chapel Hill, NC 27514, USA \\ ${ }^{\mathrm{c}}$ Department of Cognitive Neuroscience, Maastricht University, Oxfordlaan 55, 6229 EV Maastricht, The Netherlands \\ ${ }^{\mathrm{d}}$ Faculty of Medicine and Life Sciences, Hasselt University, Agoralaan, Building C, 3590 Diepenbeek, Belgium \\ e Department of Psychology, KU Leuven, PO Box 3726, 3000 Leuven, Belgium \\ ${ }^{\mathrm{f}}$ Department of Welfare, Public Health and Family, Flemish Government, PO Box 30, 1030 Brussels, Belgium
}

\section{A R T I C L E I N F O}

\section{Article history:}

Accepted 3 December 2014

\section{Keywords:}

Developmental origins of health and disease (DOBHAD)

Anxiety

Infant

Event-related potential (ERP)

Multimodal processing

Emotion perception

\begin{abstract}
A B S T R A C T
The ability to read emotional expressions from human face and voice is an important skill in our day-today interactions with others. How this ability develops may be influenced by atypical experiences early in life. Here, we investigated multimodal processing of fearful and happy face/voice pairs in 9-month-olds prenatally exposed to maternal anxiety, using event-related potentials (ERPs). Infants were presented with emotional vocalisations (happy/fearful) preceded by emotional facial expressions (happy/fearful). The results revealed larger P350 amplitudes in response to fearful vocalisations when infants had been exposed to higher levels of anxiety, regardless of the type of visual prime, which may indicate increased attention to fearful vocalisations. A trend for a positive association between P150 amplitudes and maternal anxiety scores during pregnancy may suggest these infants are more easily aroused by and extract features more thoroughly from fearful vocalisations as well. These findings are compatible with the hypothesis that prenatal exposure to maternal anxiety is related to more extensive processing of fearrelated stimuli.
\end{abstract}

(c) 2014 Elsevier Inc. All rights reserved.

\section{Introduction}

The ability to read each other's emotional expression is an important skill in our day-to-day interactions with others. In most social situations, emotional information can be inferred from more than one modality simultaneously, such as from both the face and the voice of the other person. Previous research has found that emotional information from one modality, such as a face, may influence how emotional information from another modality, such as a voice, is processed and perceived. For example, de Gelder and Vroomen (2000) and de Gelder, Pourtois, and Weiskrantz (2002) found that in adults, both recognition and judgement of emotions from voice can be modulated by consciously as well as uncon-

Abbreviations: GA, gestational age; PELS, prenatal early life stress study; STAI, Spielberger State-Trait Anxiety Inventory; SCL, symptom checklist-90; Nc, negative component; Pc, positive component.

* Corresponding author at: Department of Psychology, Tilburg University, PO Box 90153, 5000 LE Tilburg, The Netherlands. Fax: +31 (0)13 4662067.

E-mail addresses: r.a.otte@tilburguniversity.edu (R.A. Otte), franc.donkers@ maastrichtuniversity.nl (F.C.L. Donkers), m.braeken@tilburguniversity.edu (M.A.K. A. Braeken), bea.vdnbergh@tilburguniversity.edu (B.R.H. Van den Bergh). sciously recognised emotion in the corresponding face. Developmental studies have shown that in infants, too, emotional faces may affect processing of emotional voices and the other way around (e.g. Flom \& Bahrick, 2007; Grossmann, Striano, \& Friederici, 2006; Walker-Andrews \& Lennon, 1991; for a review, see Grossmann, 2010). What has not been studied, however, is the potential effect of early life influences on the ability to detect and process emotional audiovisual information. If the development of the auditory or the visual system is altered or even disrupted, for instance by atypical maternal psychological mood during pregnancy, this may change how information is processed for both modalities. The goal of the current study, therefore, was to examine whether early life experiences, in the form of prenatal exposure to maternal anxiety, influence the ability to process emotional information from face and voice.

Over the last decades, evidence has become available that exposure to atypical or adverse environmental factors during gestation may modulate the programming of genetically based neural architecture of the brain, thereby changing the developmental pathway of neurocognitive processes (part of the field 'developmental origins of behaviour, health and disease (DOBHaD)'; see 
e.g. Gluckman, Hanson, \& Buklijas, 2010; Räikkönen, Seckl, Pesonen, Simons, \& Van den Bergh, 2011, and Van den Bergh, 2011 for reviews). The DOBHAD hypothesis encompasses the short- and long-term consequences of the conditions of the developmental environment for health and disease risk (Gluckman et al., 2010). For instance, iron deficiency during life in the womb, as inferred from serum ferritin concentrations at birth, has been associated with impaired auditory recognition memory in newborns and infants, possibly due to disruptions of the myelination process (Beard, 2008; Georgieff, 2008; Georgieff, Wewerka, Nelson, \& deRegnier, 2002; Nelson et al., 2000; Siddappa et al., 2004). In the same vein, the psychological status of the mother during pregnancy may influence offspring neurocognitive development. In evidence of this, associations have been found between prenatal exposure to maternal stress, depression, and anxiety, and alterations in infant, child, and adolescent neurocognition. As an example, in a previous study, we found that maternal anxiety during pregnancy influences processing of rapidly presented sound sequences in both 2-month-old (Otte, Braeken, Winkler, \& Van den Bergh, 2011) and 9-month-old infants (van den Heuvel, Donkers, Winkler, Otte, \& Van den Bergh, 2014). Also, Weikum, Oberlander, Hensch, \& Werker (2012) described that prenatal exposure to maternal depressed mood shifted developmental milestones in infant speech perception. (For other examples, see e.g. Charil, LaPlante, Vaillancourt, \& King, 2010; Entringer, Buss, \& Wadhwa, 2010; Mennes, Stiers, Lagae, \& Van den Bergh, 2006; Sandman, Davis, Buss, \& Glynn, 2011; Van den Bergh, 2011; Van den Bergh, Mulder, Mennes, \& Glover, 2005). There is as yet no full understanding of the exact mechanisms underlying these findings, although a body of literature suggests that the hormone cortisol and the placental enzyme $11 \beta$-HSD2 may play a role (e.g. Harris \& Seckl, 2011; Monk, Fitelson, \& Werner, 2011; Wyrwoll, Holmes, \& Seckl, 2011). Also, recent findings indicate that epigenetic dysregulation is an important underlying mechanism (Gillies, Virdee, McArthur, \& Dalley, 2014; Graignic-Philippe, Dayan, Chokron, Jacquet, \& Tordjman, 2014; Hompes et al., 2013; O'Donnell et al., 2012; Van den Bergh, 2011). Here, we pose the question whether altered multimodal processing of emotional stimuli can also be related to in utero exposure to maternal anxiety.

To answer this question, we studied multimodal processing of emotional information in 9-month-old infants by means of event-related potentials (ERPs). Until now, behavioural measures have most often been used to study both multimodal processing of emotional information in infants, and infant neurocognitive outcome following prenatal exposure to maternal anxiety. ERPs, however, have a number of advantages over behavioural measures. First, ERPs provide a continuous measure of information processing from stimulus exposure to until after elaboration of the response, which allows for assessment of which stage(s) of processing are affected by a specific experimental manipulation (Luck, 2005). Second, ERPs have a very high temporal resolution, and therefore the timing of neurocognitive processes can be studied with millisecond precision (Luck, 2005). Third, ERPs can be recorded in the absence of a behavioural response (Nelson \& Bloom, 1997), even for unattended stimuli (Sussman, 2007). This makes them quite suitable for studying actual brain functioning in neonates and infants, instead of making inferences about neurocognitive functioning based on behavioural measures.

The paradigm for the current study was based on an ERP study by Grossmann et al. (2006), who investigated in 7-month-old infants the processing of angry and happy vocalisations following the presentation of an emotionally congruent or incongruent facial expression (prime). The authors found that the emotionally incongruent condition elicited a larger auditory Negative component (Nc) around $500 \mathrm{~ms}$ post-stimulus. In contrast, the emotionally congruent condition elicited a larger auditory Positive component
(Pc) approximately $800 \mathrm{~ms}$ after stimulus onset. Grossmann et al. (2006) concluded that the attenuation of the Nc and enhancement of the later Pc for the congruent condition reflected recognition of the familiar/expected face/voice pairs, and that the infants had thus recognised and processed emotions from both modalities.

To our knowledge the study by Grossmann et al. (2006) is the only ERP study with infants in which the processing of emotional auditory stimuli following an emotional visual prime is investigated, instead of the other way around. However, since the auditory system develops earlier than the visual system (Anderson \& Thomason, 2013; Anderson et al., 2001), from a developmental perspective, emotional vocalisations may be just as relevant as facial expressions in the first months of life. Thus, to supplement the existing literature with data on the processing of emotional vocalisations in infancy, in the current study (and in our twinpaper Otte et al., 2015) we focused on effects of emotional facial expressions on the processing of emotional auditory stimuli (in the context of prenatal exposure to maternal anxiety).

Following Grossmann et al. (2006) we used both a positive and a negative emotion (happiness and fear), and investigated the processing of auditory vocalisations following an emotional (happy/ fearful) visual prime. In contrast to Grossmann et al. (2006), fear instead of anger was used as the negative emotion. We had several reasons for this. First, research has found that (1) exposure to maternal anxiety is associated with more anxiety in the offspring (Lupien, McEwen, Gunnar, \& Heim, 2009; O'Connor et al., 2002; Van den Bergh \& Marcoen, 2004); (2) individuals with high state anxiety respond stronger to fearful stimuli (Bishop, Duncan, \& Lawrence, 2004); and (3) high trait anxiety has been related to altered processing of emotional information from face and voice (Koizumi et al., 2011). Studying responses to fearful stimuli may yield insights into how these findings relate to each other. Second, infants display increased attention to fearful stimuli, at least from 7 months old onwards (Kotsoni, de Haan, \& Johnson, 2001; Montague \& Walker-Andrews, 2001; Peltola, Leppänen, Mäki, \& Hietanen, 2009).

As this study represented the first effort to relate prenatal exposure to maternal anxiety to processing of multimodal emotional information, our hypotheses were of an exploratory nature. We hypothesised that higher levels of maternal anxiety during pregnancy (1) would be associated with larger responses to fearful auditory stimuli; and (2) would most strongly affect responses to auditory stimuli which had been preceded by visual stimuli conveying the same (versus a different) emotion, reflecting a relationship between prenatal exposure to maternal anxiety and multimodal processing of emotional information. Leaving from the paper by Grossmann et al. (2006), we were interested in potential modulations of the auditory Nc and Pc by prenatal exposure to maternal anxiety. Because of the exploratory nature of the study and the fact that infants in our study were about 2 months older than the infants in the study by Grossmann et al. (2006), we also examined the earlier occurring auditory ERP components P150, N250 and P350.

\section{Methods}

\subsection{Subjects}

Subjects were 82 infants (one pair of twins) and their mothers from a normal (i.e. non-clinical) population who have been taking part in a longitudinal study on prenatal early life stress (PELS project). The study was approved by the Medical Ethical Committee of St. Elizabeth Hospital in Tilburg, The Netherlands. Informed consent was obtained from all mothers and fathers in accordance with the Declaration of Helsinki. Detailed information on the cohort and its recruitment has been described previously in Otte et al. (2013). 
In short, the cohort consists of 190 women - and their partner and child - who have been recruited during pregnancy, either before 15 weeks gestational age (GA; $N=178)$ or between week 16 and $22(N=12)$ of gestation from a general hospital and four midwives' practices in Tilburg, The Netherlands. Women were followed up three times during their pregnancies (measurement waves T1, T2 and T3, respectively) and were invited to the lab for postpartum observations both 2-4 months (T4) and 9-11 months (T5) after giving birth. Here, we report the results from infants measured at T5; data collected at T4 have been discussed elsewhere (Otte et al., 2013; van den Heuvel et al., submitted for publication).

At T5, 147 of the original 190 women came in for testing with their infant (one pair of twins). Forty-three women did not participate in this measurement wave, because of drop out before T5 $(N=32)$, because they could not be reached in time (6), they were $(N=1)$ or their infant was $(N=2)$ too ill, they had miscarried $(N=1)$ around T2 or their infant had passed away $(N=1)$. Three of the 147 mothers had delivered prematurely, 1 mother had delivered a baby small for GA (e.g. birth weight $<2500 \mathrm{~g}$ at term delivery), and 2 mothers had been under treatment for psychological problems at T1 (depression and personality problems, respectively), potentially influencing the questionnaire data collected at that time. Data for infants of these mothers $(N=6)$ were excluded from analysis beforehand. Data for an additional 60 of the remaining 142 infants were later excluded because of too little remaining data after removing invalid trials (e.g. with movement artefacts, and during which the infant had not looked at the stimulus; $N=33$ ), fussiness $(N=13)$, and technical problems (e.g. severe problems with mastoids; $N=14)$. This attrition rate $(42.2 \%)$ is similar to other infant ERP studies (DeBoer, Scott, \& Nelson, 2007). All infants were healthy and had passed a screening test for hearing impairments (evoked otoacoustic emission), performed by a nurse from the infant health care clinic, between the 4th and 7th day after birth. Demographical statistics of the infants, and their mothers, are given in Table 1.

\subsection{Predictor}

Maternal anxiety during pregnancy was measured before 15 weeks GA, with Dutch versions of the anxiety subscales of the Spielberger State-Trait Anxiety Inventory (STAI; Ploeg, Defares, \& Spielberger, 1980; Spielberger, Gorsuch, \& Lushene, 1970), and the Symptom Checklist-90-R (SCL; Arrindell \& Ettema, 2003; Derogatis \& Cleary, 1977; Derogatis, Lipman, \& Covi, 1973). We specifically focused on early pregnancy, because previous research associated exposure during early rather than late gestation with specific changes in offspring cognitive functions as measured with behavioural (i.e. neuropsychological) as well as with physiological (i.e., ERP and fMRI) measures (Malaspina et al., 2008; Mennes, Van den Bergh, Lagae, \& Stiers, 2009; Mennes et al., 2006; Van den Bergh et al., 2006).

\subsubsection{Anxiety subscale from the STAI}

The STAI is a self-report questionnaire with the subscales "Trait" and "State" Anxiety. Both scales consist of 20 items which can be rated on a Lickert scale from 1 (not at all/almost never) to 4 (very much/almost always). Whereas trait anxiety refers to a disposition or proneness to react with anxiety, the state anxiety subscale provides a valid measure of the intensity of transitory anxiety in response to real life stress (Spielberger, 1975). Therefore, maternal state (and not trait) anxiety was used as a measure of prenatal maternal anxiety. The subscale's Cronbach's $\alpha$ was .902 in the current study. Examples of items on the State scale are: "I feel secure", "I am worried" and "I feel indecisive".

\subsubsection{Anxiety subscale from the SCL-90-R}

The SCL, too, is a self-report instrument. The questionnaire quantifies current psychopathological symptoms in terms of nine primary constructs, of which anxiety is one. The anxiety subscale measures current feelings of more generalised anxiety, with complaints related to increased vegetative arousal, general symptoms such as nervousness, more specific symptoms such as panic attacks, and cognitive symptoms such as fearful thoughts (Arrindell \& Ettema, 2003; Derogatis \& Cleary, 1977). The 10 items are rated on a 5-point Lickert scale, ranging from 0 (not at all) to 4 (extremely). Cronbach's $\alpha$ for this subscale was .861 in the current study. Examples of items are: "Heart pounding or racing", "Trembling", and "Spells of terror or panic".

\subsubsection{Postpartum anxiety}

To control for postpartum anxiety, the state anxiety subscales of both the STAI and the SCL were also administered at T5, at about the same time the mother and her infant were invited to the lab for the T5 observations. Anxiety data were unavailable for 4 mothers at $\mathrm{T} 1$ because of recruitment after this first data collection wave. In addition, 17 mothers did not return the questionnaire at T5. The missing anxiety sum scores were imputed, ${ }^{1}$ separately per questionnaire, by means of the Expectation-Maximization method as implemented in IBM SPSS Statistics 19.0.Variables used for the EM were anxiety sum scores measured at least two other time points; e.g. when the anxiety sum score was missing for T1, the available anxiety sum scores from the same questionnaire (so STAI data for imputing STAI data, and SCL data for imputing SCL data) measured from T2, T3, T4 and/or T5 were used to estimate the missing score.

\subsection{Stimuli}

\subsubsection{Visual stimuli}

Visual stimuli were 18 colour photos of 9 Caucasian women in frontal view, each expressing both happiness and fear. Only female and Caucasian identities had been chosen so as to avoid any sex or ethnicity differences from influencing the infant ERPs (e.g. see Ramsey, Langlois, \& Marti, 2005; Vogel, Monesson, \& Scott, 2012). The emotional faces had been cut out from their original background and pasted onto a black background. Four identities were taken from the validated NimStim Face Stimulus Set (http://www.macbrain.org/resources.htm ${ }^{2}$ ). Their fearful and happy expression had been recognised at least $75 \%$ of the time in the validation study. Examples of a typical happy and fearful expression from the NimStim dataset can be found in Fig. 1A and B, respectively.

An additional 5 identities were included from a database with emotional facial stimuli from the Cognitive and Affective Neurosciences Laboratory at Tilburg University, The Netherlands. This was done because we wanted to include as many identities as possible to minimise the possibility that potential effects would be due to the identity itself, and the NimStim set did not offer more than 4 female Caucasian identities whose fearful and happy expression survived the validation process. The stimuli from the Tilburg database had been validated in a pilot study in which they were rated for emotion (fear, happiness, anger, neutral, surprise, sadness and disgust), intensity (scale from 1 to 5 ) and positive/negative affect (scale from 1 to 5 ) by at least 8 participants. For the 5 identities used for this study both the emotion fear and happiness were cor-

\footnotetext{
${ }^{1}$ Please note that we imputed the missing sum scores, not the missing item scores. 2 Development of the MacBrain Face Stimulus Set was overseen by Nim Tottenham and supported by the John D. and Catherine T. MacArthur Foundation Research Network on Early Experience and Brain Development. Please contact Nim Tottenham at tott0006@tc.umn.edu for more information concerning the stimulus set.
} 
Table 1

Sample characteristics of participating women and their infants.

\begin{tabular}{|c|c|c|c|c|c|}
\hline & & & $N$ & $\%$ & $M(\mathrm{SD})$ \\
\hline \multirow[t]{22}{*}{ Mothers } & & & 81 & & \\
\hline & Age at T1 (years) & & & & $32.39(3.80)$ \\
\hline & State anxiety at $\mathrm{T} 1$ & & & & $32.82(8.89)$ \\
\hline & State anxiety at $\mathrm{T} 5$ & & & & $31.92(7.66)$ \\
\hline & Marital status & Married & 45 & 55.6 & \\
\hline & & Cohabiting & 37 & 43.2 & \\
\hline & & Single & 1 & 1.2 & \\
\hline & Educational level & Primary or secondary & 7 & 8.0 & \\
\hline & & General vocational training & 18 & 20.7 & \\
\hline & & Higher vocational training & 42 & 48.3 & \\
\hline & & University degree or higher & 20 & 23.0 & \\
\hline & Family income (monthly, in $€$ ) & $<2100$ & 3 & 3.4 & \\
\hline & & $2200-3600$ & 19 & 21.8 & \\
\hline & & $>3600$ & 62 & 71.3 & \\
\hline & & Don't want to disclose & 3 & 3.4 & \\
\hline & Primigravida & & 35 & 40.2 & \\
\hline & Smoking during pregnancy & & 3 & 3.4 & \\
\hline & Drinking during pregnancy & & 7 & 8.0 & \\
\hline & Has been treated in the past for & Depression & 8 & 9.2 & \\
\hline & & Anxiety problems & 4 & 4.6 & \\
\hline & & Personality problems & 2 & 2.3 & \\
\hline & & PDD-NOS & 1 & 1.1 & \\
\hline \multirow[t]{6}{*}{ Infants } & & & 82 & & \\
\hline & Sex & Boy & 38 & 46.3 & \\
\hline & & Girl & 44 & 53.7 & \\
\hline & Birth weight (grams) & & & & 3474 (569) \\
\hline & GA at birth (weeks) & & & & $39.9(1.3)$ \\
\hline & Age at T5 (days) & & & & $303.1(14.1)$ \\
\hline
\end{tabular}

GA = gestational age; PPD-NOS = Pervasive Developmental Disorder-Not Otherwise Specified.

${ }^{\text {a }}$ From 1 glass of wine or liquor during the whole pregnancy to maximally 3 glasses a month.

rectly recognised by the raters $80 \%$ of the time or more. In Fig. $1 \mathrm{C}$ and $\mathrm{D}$ examples of a happy and fearful face from the Tilburg dataset can be found.

\subsubsection{Auditory stimuli}

Auditory stimuli were voice recordings of six women expressing fear and happiness with non-verbal vocalisations. We chose to use non-verbal vocalisations as to minimise automatic semantic or verbal processing (Van den Stock, Grèze, \& de Gelder, 2008). Because not all vocalisations had been recognised well enough in the validation study (see below), for two of the identities we only used the fearful vocalisation, for two of them we only used the happy vocalisation and for two of them we used vocalisations of both emotions, resulting in 4 fearful and 4 happy auditory stimuli. These auditory stimuli were provided by the Cognitive and Affective Neurosciences Laboratory at Tilburg University, too, and were recorded, processed and validated as described by Van den Stock et al. (2008). In short, semi-professional actors were asked to make a frightened (e.g. sudden intake of breath) or happy (e.g. laughter) sound, based on a specific script describing situations such as an attack by a robber. The $800 \mathrm{~ms}$ audio recordings were made at a $44.1 \mathrm{kHz}$ sampling rate and were shortened to $500 \mathrm{~ms}$ for the experiment described here. The sounds were validated in a pilot session by 15 participants, who were instructed to categorise as accurately and as fast as possible the emotion expressed by the voice (fear or happiness). All stimuli used here had been correctly recognised $80 \%$ of the time or more.

Neither the visual nor the auditory stimuli were controlled for low level perceptual features. However, since for each stimulus category, 6/9 different exemplars were used, the likelihood should be decreased that potential effects may be due to low-level fea- tures unconnected to the emotional content (i.e., some low-level features serve as cues for the given emotional content).

\subsection{Procedure}

Each of the 18 visual stimuli was paired with both the fearful and happy auditory stimuli, resulting in 144 face/voice compounds and four experimental conditions: happy face-happy voice $(\mathrm{HH})$, fearful face-fearful voice (FF), happy face-fearful voice (HF), and fearful face-happy voice (FH). The compounds were presented twice during the experiment (288 trials), divided in four blocks of 72 stimuli, each. The presentation order within each of the blocks was randomised, and the order between the blocks was counter-balanced. The blocks were presented with small breaks in between as needed.

During stimulus presentation the infant was seated on its parent's lap in a dimly lit and sound-attenuated room of the Tilburg University Babylab. The parent-infant dyad were seated behind a desk with a computer screen (CRT VGA, 21 in., $1280 \times 1024$, $100 \mathrm{~Hz}$ ) at a distance of approximately $70 \mathrm{~cm}$ from the infant's eyes. The visual stimuli measured $18.5 \times 22.5 \mathrm{~cm}$ and the horizontal and vertical visual angles were $7.53^{\circ}$ and $9.13^{\circ}$, respectively. Auditory stimuli were presented through speakers positioned on either side of the screen, and at a distance of approximately $90 \mathrm{~cm}$ from the infant's head. To prevent the parent from influencing the infant's ERP responses by unconsciously reacting to the stimuli, he or she was wearing head phones through which classical music was playing. ${ }^{3}$ Two cameras filmed the experimental ses-

\footnotetext{
${ }^{3}$ Since for this experiment we were interested in the infants' responses to emotional vocalisations, we did not prevent the mothers from seeing (and potentially responding to) the visual stimuli.
} 
(A)

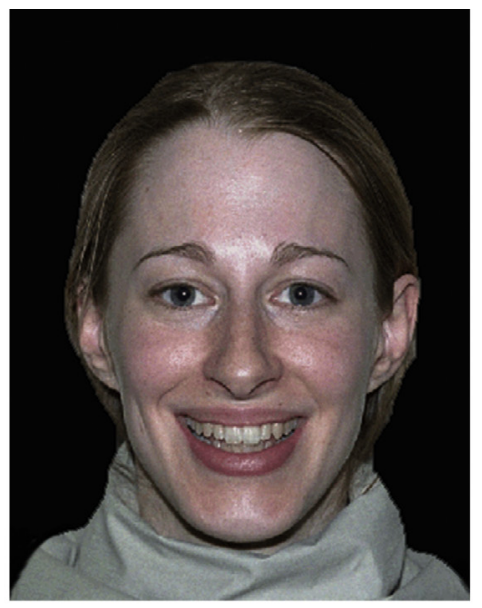

(C)

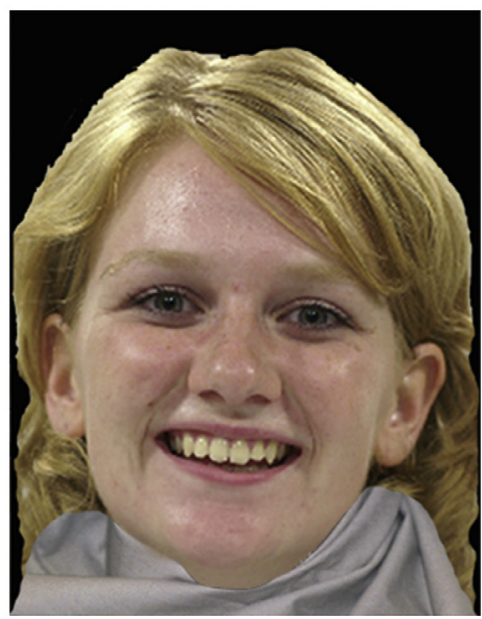

(B)

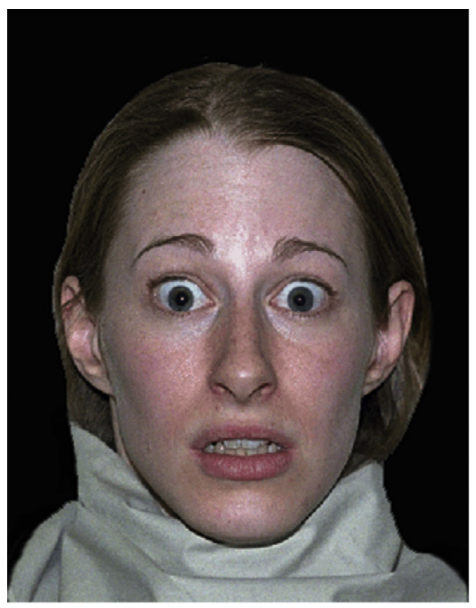

(D)

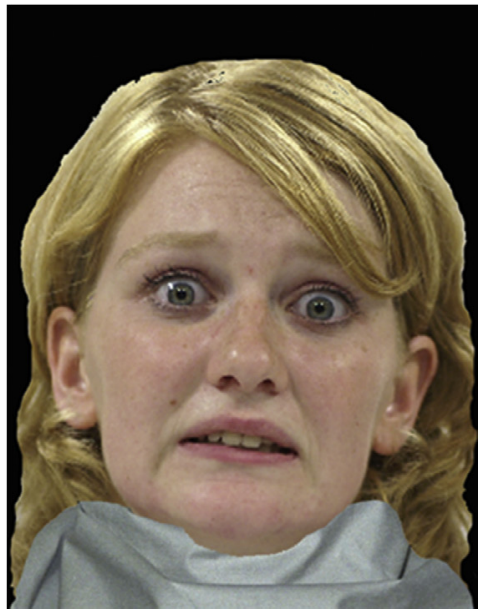

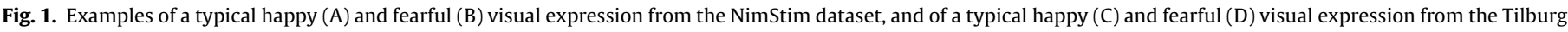
dataset.

sion and these data were later used to code whether the infant had looked at a specific trial or not (see also Section 2.5).

Each stimulus block started with the sound of a laughing baby, and the presentation of a red dot growing bigger and smaller in the centre of the computer screen to attract the infant's attention. When the infant was looking at the screen, the experimenter started the first trial. Each trial lasted $1400 \mathrm{~ms}$ and started with the presentation of a visual stimulus that lasted $900 \mathrm{~ms}$, after which an auditory stimulus was presented. The visual stimulus remained in place until the auditory stimulus was played out (after $500 \mathrm{~ms}$ ). Each trial was followed by an inter-trial interval with variable duration (between 600 and $1000 \mathrm{~ms}$ ) to reduce temporal predictability. During this interval, the screen was black. When an infant looked away from the screen, the experimenter tried to recapture the infant's attention by presenting an attractive moving figure in the centre of the screen. As soon as the infant was looking at the screen again, the experiment continued. The experiment was concluded either after all 288 stimuli had been presented or if the infant became too fussy to continue.

\subsection{Data acquisition and analysis}

EEG was recorded with BioSemi ActiveTwo amplifiers (BioSemi, Amsterdam, The Netherlands) with a sampling rate of $512 \mathrm{~Hz} .{ }^{4}$

\footnotetext{
${ }^{4}$ On-line filtering options in the BioSemi software are set automatically based upon the sampling rate. See http://www.biosemi.com/faq/adjustfilter.htm and http:// www.biosemi.com/faq/adjustsamplerate.htm.
}

Infants wore head caps with 64 electrode locations positioned according to the revised version of the International 10-20 system. The standard BioSemi reference (CMS-DRL) was used (see www.biosemi.com/faq/cms\&drl.htm for details) and two additional electrodes were placed both on the left and right mastoid. Off-line, these were mathematically combined to produce an average mastoids reference derivation (Luck, 2005).

Before the data were processed further, independent raters inspected the data and scored per infant per trial whether or not he or she had indeed looked at the visual stimulus. All trials were scored by 2 different raters (there were 4 raters in total) and these scorings were afterwards compared. Agreement between raters lay between $81 \%$ and $99 \%$ and was $95 \%$ on average. Whenever scorings differed, the trials concerned were re-inspected and scored again. If there was still doubt about whether the infant had actually seen the stimulus, the trial was excluded. Trials during which an infant was crying were also excluded. Only EEG signals from trials during which the infant was looking at the stimulus were used for data analysis.

The EEG data were analysed using BrainVision Analyzer software (BrainProducts, Munich, Germany). The continuous EEG signals were filtered off-line, with a $50 \mathrm{~Hz}$ notch filter (to make sure all line noise would be removed) and a $0.1-20 \mathrm{~Hz}$ band-pass filter (slope $24 \mathrm{~dB}$ ). The signals were then segmented into $1000 \mathrm{~ms}$-long epochs, time-locked to the onset of the auditory stimulus. The $200 \mathrm{~ms}$ before auditory stimulus onset were used as the baseline. To make sure that this auditory baseline did not differ between happy and fearful facial expressions, we tested it. We did this by 
first calculating - per subject, per condition - the average amplitude in the auditory baseline period ( -200 to $0 \mathrm{~ms}$ before voice onset), using the $200 \mathrm{~ms}$ before face onset (so -1100 to $-900 \mathrm{~ms}$ before voice onset) as baseline. Then, we ran a 4-way ANOVA with the factor "Condition" (4 levels: FH, FF, HF and HH) to test whether there were differences between the 4 conditions in the auditory baseline period. The result $(F(3,267)=2.192, p=.089)$ showed no differences between the four conditions, and we concluded the $200 \mathrm{~ms}$ before onset of the auditory stimulus were suitable for use as the baseline.

The epochs were averaged separately for each of the four conditions (FF, HH, HF, FH). Epochs with sample-to-sample voltage steps exceeding $80 \mu \mathrm{V}$ were excluded, as were epochs with amplitudes exceeding $150 \mu \mathrm{V}$ in any $200 \mathrm{~ms}$-long window within the whole epoch, and those where the amplitude range in any 100 -ms window, was below $0.5 \mu \mathrm{V}$ (i.e., flat lines). Data from infants with less than 14 acceptable responses for any one of the four conditions were removed from further analysis (33 infants, see also Section 2.1). The average number of available trials per infant was 36.5 for condition FF ( $S D=12.6), 37.5$ for $\mathrm{HH}(S D=11.9), 36.6$ for $\mathrm{HF}(S D=11.7)$, and 37.1 for $\mathrm{FH}(S D=12.7)$.

Time windows for analysis were selected based on visual inspection of the grand average waveform for all responses combined at electrode sites F3, Fz, F4, C3, Cz, C4 (see our related twin paper Otte, Donkers, Braeken, and Van den Bergh (2015) for the morphology of the overall ERP response), where responses were largest. The following windows, each centred around a peak in the grand average waveform, were chosen: 120-200 ms, 200$260 \mathrm{~ms}, 290-430 \mathrm{~ms}, 380-540 \mathrm{~ms}$, and $620-680 \mathrm{~ms}$ post-stimulus (from this point on referred to as P150, N250, P350, N450, and $\mathrm{P} 650$, respectively). The $\mathrm{N} 450$ and $\mathrm{P} 650$ can be regarded as the approximate equivalent of the Nc and Pc, respectively, described by Grossmann et al. (2006). Compared to their components, the peak latency of the N450 and P650 is somewhat earlier, which may be due to maturational changes. Together with the $\mathrm{Nc} / \mathrm{N} 450$, the P150, N250 and P350 correspond to the infant P150-N250P350-N450/Nc ERP pattern in response to auditory stimuli described by Kushnerenko et al. (2002), who found that this pattern is already identifiable in auditory ERPs from birthSince Nc and Pc usually refer to visual components, in the current paper we refer to them as $\mathrm{N} 450$ and $\mathrm{P} 650$, respectively.

To answer our research question, average amplitudes measured from the above mentioned windows were analysed by means of a $6 \times 2 \times 2$ repeated measures ANCOVAs design (run in IBM SPSS 19.0), separately for each window. Within-subjects factors were "Electrode" (F3, Fz, F4, C3, Cz, C4), "Visual Prime" (fearful, happy), and "Auditory Emotion" (fearful, happy). Continuous predictor variables ('covariates' in SPSS's ANCOVA) were state anxiety measured with the STAI (StatAnx), and more generalised anxiety measured with the SCL (GenAnx). Separate analyses were run for both predictor variables. We chose not to enter StateAnx and GenAnx as between-subjects factors (e.g. high versus low anxiety), because dichotomising the variables would result in groups of different sizes, and because previous research has shown that dichotomisation of a continuous variable can lead to problems such as loss of effect size, power and/or information about individual differences (Altman \& Royston, 2006; MacCallum, Zhang, Preacher, \& Rucker, 2002; Royston, Altman, \& Sauerbrei, 2006). Post-hoc tests were run to further investigate any significant main/interaction effect. We were especially interested in effects of priming by the visual stimulus (Visual Prime) on the auditory stimulus (Auditory Emotion) in the context of exposure to anxiety, because this factor represents effects of the visually presented emotion on the processing of the emotional sounds. However, as our research question and hypotheses were of an exploratory nature, we ran post-hoc tests for all effects including StateAnx and GenAnx.
Previous research has shown that several factors may affect and/or confound both infant ERPs and the association between prenatal exposure to atypical cues and infant cognitive outcome (e.g. see Fellman et al., 2004; Heron, O'Connor, Evans, Golding, \& Glover, 2004; Kodituwakku, 2007; Kushnerenko et al., 2002; Lavoie, Robaey, Stauder, Glorieux, \& Lefebvre, 1998; Rodriguez \& Bohlin, 2005; Streissguth, Barr, \& Martin, 1983). Therefore, we controlled for the following factors by adding them, one at a time, as covariate to the analyses: Maternal alcohol intake during pregnancy (continuous) and postpartum anxiety; and infant sex, birth weight (controlled for gestational age), gestational age and age at testing. The continuous factors were first correlated to the ERP data and only added when significant correlations were found. The dichotomous covariates were added as between-subjects factor to the analyses. As only three mothers had smoked during pregnancy, we controlled for this factor by running the analyses with and without infants from smoking mothers, and then examining whether effects remained the same. Greenhouse-Geisser correction was used where necessary and the $\varepsilon$ correction factor is given, together with the partial $\eta^{2}$ effect size in Table 2.

\section{Results}

The grand-averaged auditory waveform for responses to all four stimulus types (FF, HH, HF, FH) combined can be found in our twin paper (see Otte et al., 2015). This figure was used to select time windows for the statistical analyses (see "Methods" Section 2.5). As there were no significant effects involving StatAnx only findings for GenAnx will be reported here. For investigating our hypotheses, all main and interaction effects including the latter factor will be interpreted, including trends (see Table 2 for statistical information). Significant results involving other factors can also be found in Table 2.

\subsection{Results for GenAnx}

\subsubsection{Results for the P150}

There was an interaction effect trending towards being significant between Auditory Emotion and GenAnx. As can be seen in the plot of the interaction in Fig. 2A, it was caused by a positive association between GenAnx and fearful vocalisations (larger maternal state anxiety scores associated with larger positive amplitudes) versus a negative association between GenAnx and happy vocalisation (larger maternal state anxiety scores associated with smaller positive amplitudes). Analyses of simple effects (i.e. between GenAnx and fearful vocalisations and between GenAnx and happy vocalisations) showed that the neither of these were significant (fearful vocalisations: $\beta=.139, p=.309$. Happy vocalisations: $\beta=-.088, p=.528$ ). The interaction effect remained intact after controlling for the covariates described in the methods Section 2.5.

There were no associations between Visual Prime and GenAnx.

\subsubsection{Results for the $N 250$}

The analysis yielded no effects with the factor GenAnx.

\subsubsection{Results for the $P 350$}

There was a significant interaction effect between Auditory Emotion and GenAnx, which was caused by a positive association between GenAnx and fearful vocalisations (larger maternal state anxiety scores associated with larger positive amplitudes versus a negative association between GenAnx and happy vocalisation with larger maternal state anxiety scores associated with smaller positive amplitudes) - see a plot of the interaction in Fig. 2B. Simple effects analyses between GenAnx and fearful vocalisations and 
Table 2

Significant main and interaction effects for GenAnx.

\begin{tabular}{|c|c|c|c|c|c|}
\hline Factor(s) & $d f$ & $F$ & $p$ & $\varepsilon$ & $\eta^{2}$ \\
\hline \multicolumn{6}{|l|}{ 120-200 ms latency range } \\
\hline Electrode & $(5,400)$ & 2.750 & .048 & \multirow[t]{4}{*}{.56} & .033 \\
\hline Visual prime & $(1,80)$ & 3.896 & .052 & & .046 \\
\hline Auditory Emotion $\times$ GenAnx & $(1,80)$ & 3.568 & .063 & & .043 \\
\hline Visual prime $\times$ Auditory Emotion & $(1,80)$ & 4.215 & .043 & & .050 \\
\hline \multicolumn{6}{|l|}{ 200-260 ms latency range } \\
\hline Electrode & $(5,400)$ & 3.504 & .019 & \multirow[t]{2}{*}{.55} & .042 \\
\hline Visual prime & $(1,80)$ & 6.408 & .013 & & .074 \\
\hline \multicolumn{6}{|l|}{ 290-430 ms latency range } \\
\hline Electrode & $(5,400)$ & 3.495 & .019 & \multirow[t]{3}{*}{.55} & .042 \\
\hline Visual prime & $(1,80)$ & 6.040 & .016 & & .070 \\
\hline Auditory Emotion $\times$ GenAnx & $(1,80)$ & 4.249 & .043 & & .050 \\
\hline \multicolumn{6}{|l|}{ 380-520 ms latency range } \\
\hline Electrode & $(5,400)$ & 2.245 & .092 & \multirow[t]{3}{*}{.55} & .027 \\
\hline Visual prime & $(1,80)$ & 4.132 & .045 & & .049 \\
\hline Auditory Emotion $\times$ GenAnx & $(1,80)$ & 2.941 & .090 & & .035 \\
\hline \multicolumn{6}{|l|}{ 620-680 ms latency range } \\
\hline Auditory Emotion $\times$ GenAnx & $(1,80)$ & 2.973 & .089 & & .036 \\
\hline Visual prime $\times$ Auditory Emotion $\times$ GenAnx & $(1,80)$ & 3.167 & .079 & & .038 \\
\hline
\end{tabular}
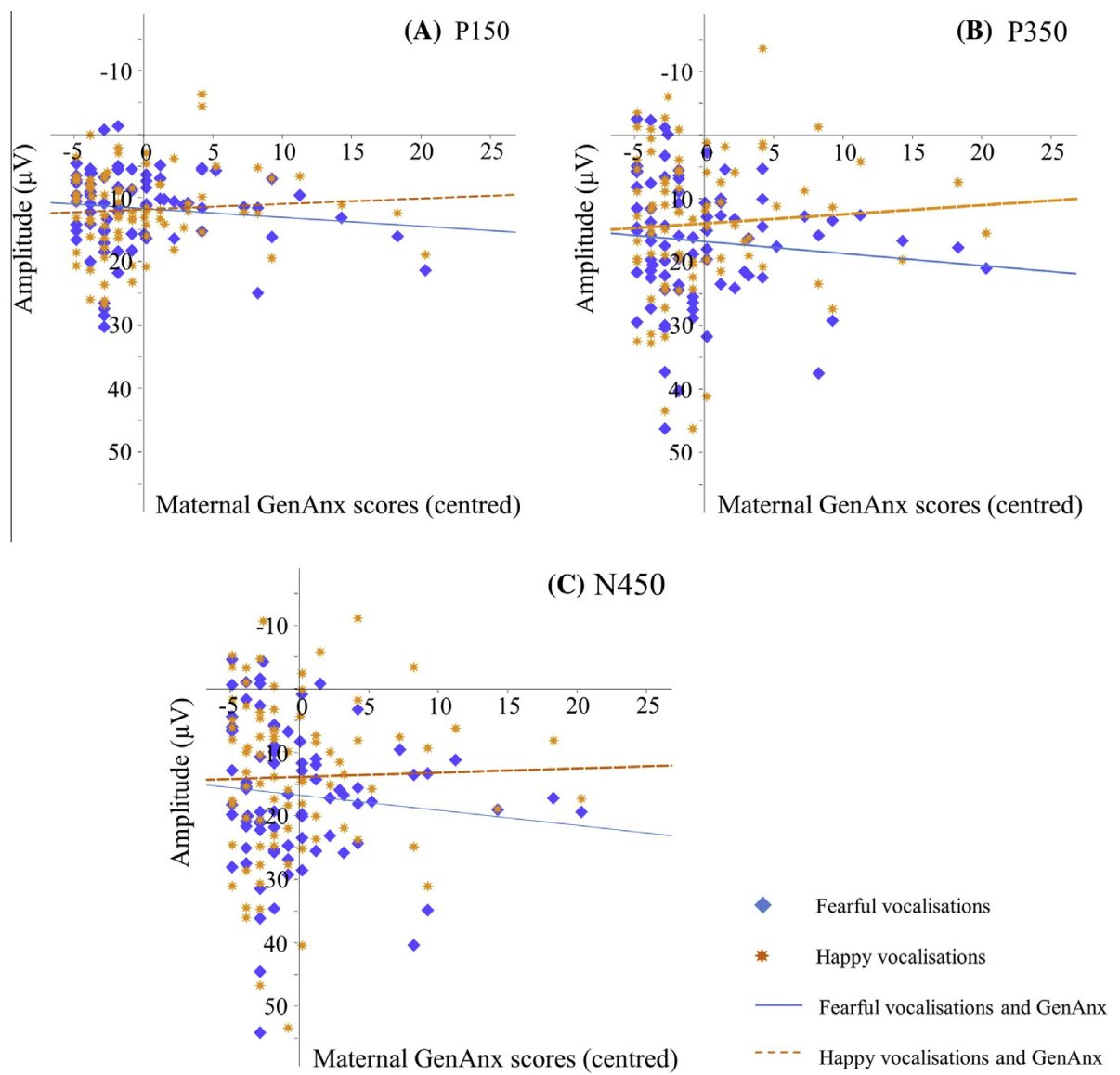

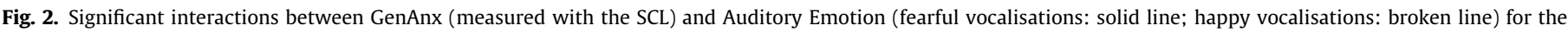
P150 (A), the P350 (B), and the N450 (C). Maternal SCL scores have been centred on the mean.

GenAnx and happy vocalisations showed neither of the two effects to be significant (fearful vocalisations: $\beta=.192, p=.367$. Happy vocalisations: $\beta=-.142, p=.563$ ). After controlling for post-partum anxiety, the interaction effect remained, but became a trend ( $p$-value increased from .043 to .052). None of the other covariates affected the interaction.

Results revealed no significant associations between Visual Prime and GenAnx.
3.1.4. Results for the $N 450$

Again, there was an interaction effect trending towards significance between Auditory Emotion and GenAnx. This was caused by the positive association between GenAnx and fearful vocalisations (larger maternal state anxiety scores associated with smaller negative, and thus more positive, amplitudes), and the negative association between GenAnx and happy vocalisations (larger maternal state anxiety scores associated with larger negative, and thus less 
positive, amplitudes), as can be seen in a plot of the interaction in Fig. 2C. Simple effects analyses were run between GenAnx and fearful vocalisations and GenAnx and happy vocalisations. Results showed neither of the two to be significant (fearful vocalisations: $\beta=.192, p=.367$. Happy vocalisations: $\beta=-.142, p=.563)$. The interaction effect remained intact after controlling for the covariates mentioned.

No associations were found between Visual Prime and GenAnx.

\subsubsection{Results for the $P 650$}

There was a 2-way interaction trending towards significance between Auditory Emotion and GenAnx, and a 3-way interaction trending towards significance between Auditory Emotion, Visual Prime, and GenAnx. Post hoc analysis on the higher order (i.e. 3way) interaction showed a negative association between GenAnx and happy vocalisations following a fearful expression (larger maternal state anxiety scores associated with smaller positive amplitudes). In contrast, for happy vocalisations following a happy expression, and for all fearful vocalisations (so both following a happy and fearful expression) a positive association with GenAnx was found (larger maternal state anxiety scores associated with larger positive amplitudes). After controlling for postpartum anxiety, both the 2- and 3-way interaction disappeared.

The analysis did not yield associations between Visual Prime and GenAnx.

\section{Discussion}

The current study investigated in 9-month-old infants the processing of emotional (fearful and happy) face/voice compounds in the context of prenatal exposure to maternal anxiety. For the P350 we found an association between the level of maternal anxiety, as measured with the SCL, and processing of emotional vocalisations. More specifically, the higher the level of maternal anxiety had been, the larger the positivity was in response to the fearful vocalisations, and the smaller in response to happy vocalisations. For the P150 and the N450 we found associations between the level of maternal anxiety, also measured with the SCL, and processing of emotional vocalisations that trended towards significance. These associations remained intact after controlling for several confounding variables, such GA and birth weight. The results confirmed our first hypothesis that higher levels of maternal anxiety during pregnancy are associated with larger responses to fearful auditory stimuli. The current study did not provide evidence for our second hypothesis that the association between exposure to maternal anxiety and responses to the audiovisual stimuli would be stronger for auditory stimuli that followed visual stimuli conveying the same (versus different) emotional information.

Our results suggest that the between-subject variability in responses to the emotional vocalisations in an audiovisual paradigm with emotional face/voice pairs can be partially explained by prenatal exposure to maternal anxiety. These findings are compatible with the DOBHAD hypothesis, which postulates that exposure to an atypical or adverse environment during pregnancy may alter the developmental pathway of the foetus and child. They are also in line with previous studies that found associations between prenatal exposure to maternal anxiety and offspring neurocognitive outcome (e.g. Bergman, Sarkar, O'Connor, Modi, \& Glover, 2007; Davis \& Sandmann, 2010; Mennes et al., 2006; Otte et al., 2011; Van den Bergh, Otte, Braeken, van den Heuvel, \& Winkler, 2012, 2013; Van den Bergh et al., 2005).

The analysis revealed a positive association between prenatal exposure to higher levels of maternal anxiety and P350 amplitudes elicited by the fearful auditory stimuli (regardless of the type of visual prime). Kushnerenko et al. (2002) suggested that infant
P350 component is a precursor to the P3a in adults and children. The P3a is thought to be involved in stimulus-driven (exogenous) attention mechanisms during task processing (e.g. Polich, 2007). For the current study, this suggests that the higher the level of maternal anxiety the infants were exposed to in the womb, the more attention they allocated to the processing of fearful sounds. In addition, for the P150 a trend towards a significant positive association with prenatal exposure to maternal anxiety was found. This infant component is proposed to be a precursor of the adult P1 (Kushnerenko et al., 2002), which shows poor sound-feature specificity, but is sensitive to arousal (Erwin \& Buchwald, 1986; Čeponiene et al., 2003). The adult P1 is therefore assumed to be involved in feature non-specific aspects of stimulus processing, like stimulus detection, facilitation/inhibition, and sensorimotor integration (Čeponienè et al., 2003). As such, the association between higher levels of maternal anxiety and larger infant P1 responses to fearful auditory stimuli may reflect a higher level of arousability (i.e. being more easily aroused) and pre-attentiveness (more thorough/elaborate feature extraction). Interpretations for both the P350 and P150 results are in line with the finding that infants who have been exposed to higher compared to lower levels of maternal anxiety display more anxiety themselves (Lupien et al., 2009; O'Connor et al., 2002; Van den Bergh \& Marcoen, 2004), and may therefore be more easily aroused by threatening stimuli (Kim et al., 2011; Rosen \& Schulkin, 1998). In this sense they are also compatible with the finding that a heightened sensitivity to threat-related information is associated with anxiety-related symptoms (Bar-Haim, 2010; Leppänen \& Nelson, 2012).

For the N450 we found smaller (negative-going) responses to the fearful stimuli. This may be because infants are generally more familiar with happy than fearful emotions (see e.g. Grossmann, 2010). Therefore, a 'negative shift' in response to the more familiar stimuli, indicating increased attention (Purhonen, KilpeläinenLees, Valkonen-Korhonen, Karhu, \& Lehtonen, 2004), may have yielded the smaller positive P350 and larger negative-going N450 amplitudes in response to the happy stimuli - and conversely the larger positive P350 and smaller negative-going N450 in response to fearful stimuli. Alternatively, it is possible that N450 responses to the fearful voice (especially when following a fearful face) originated from higher positive values, and were not strong enough to result in a larger negativity compared to the happy vocalisations.

What are possible mechanisms underlying the associations we observed in the current study? As mentioned in the introduction, a large body of literature has investigated the effects of the maternal hormone cortisol and the placental enzyme $11 \beta$-HSD2 as possible mediators between prenatal exposure to anxiety and infant (neurocognitive) outcome. During pregnancy, maternal cortisol (which may be released in response to stress and anxiety) is essential for foetal development, but excess levels may adversely affect the foetus (for reviews see e.g. Charil et al., 2010; Harris \& Seckl, 2011; Weinstock, 2008). A function of $11 \beta$-HSD2 is to convert cortisol into its inactive metabolite cortisone, protecting the foetus from over-exposure to maternal cortisol (e.g. O'Donnell et al., 2012). Maternal anxiety during pregnancy, however, has been found to down-regulate placental $11 \beta$-HSD2 activity, resulting in increased exposure of the placenta and foetus to maternal cortisol (Harris \& Seckl, 2011; O’Donnell et al., 2012; Wyrwoll et al., 2011). This may up-regulate placental corticotropin-releasing hormone (CRH), which upon reaching the foetal brain can influence neuronal differentiation and function by affecting areas rich in CRH receptors, such as para-hippocampal and limbic areas (e.g. hippocampus, amygdala; Charil et al., 2010; Weinstock, 2008). In addition, exposure to excess maternal cortisol has been associated with alterations in synaptogenesis, neurotransmitter function, and glucocorticoid receptor expression in the developing brain (De Kloet, 
Joëls, \& Holsboer, 2005; Monk et al., 2011; Oitzl, Champagne, van der Veen, \& de Kloet, 2010), which might eventually lead to altered infant neurocognitive outcome.

In addition, epigenetic changes in gene expression (i.e. potentially heritable changes without modifications in the DNA sequence) resulting from prenatal exposure to maternal anxiety - possibly in conjunction with altered levels of (placental) CRH and $11 \beta$-HSD2 and neurotransmitter activity - may underlie the results observed in this study (Gillies et al., 2014; GraignicPhilippe et al., 2014; Hompes et al., 2013; Van den Bergh, 2011). In other words, prenatal exposure to maternal anxiety might alter the typical pattern of gene expression, in that commonly expressed genes remain un-expressed or are silenced, while genes that should be silenced become expressed.

Another (complementary) mechanism potentially explaining our findings may comprise genetically inherited pathways, in that genetically heritable factors may influence both a maternal predisposition to experiencing anxiety, and offspring outcomes (Rice et al., 2010). As no genetic material has been analysed in this study, no direct evidence can be given for this explanation of the results. Indirect evidence is the association between exposure to higher levels of maternal anxiety and a trend for larger P150 amplitudes, possibly pointing to elevated levels of arousal or pre-attentiveness in the infants after exposure to threatening stimuli. However, low or complete lack of correlations between maternal and infant anxiety scores found in the current study do not support heritability of anxiety. A possible explanation of this discrepancy is that the infant anxiety measure was not sensitive enough.

Interestingly, whereas we found effects for anxiety as measured with the anxiety subscale of the SCL, we did not find effects with the state anxiety subscale of the STAI. In two former studies, however, significant associations were found in both 2- and 9-monthold infants between anxiety measured with the STAI and the processing of less informative sounds (Otte, Winkler, Braeken, \& Van den Bergh, submitted for publication; Van den Bergh et al., 2012, 2013). These differential findings are actually not very surprising, since the state anxiety subscale of the STAI and the anxiety subscale of the SCL appear to measure an overlapping, yet distinct anxiety construct (see "Methods" Section 2.2). Taking the present and former results together suggests that prenatal exposure to high levels of feelings of more generalised maternal anxiety may affect processing of familiar emotional vocalisations, while prenatal exposure to high levels of state anxiety may affect processing of sounds with low(er) information contents. However, replication or follow-up studies are needed to test whether this hypothesis holds true.

In our introduction we suggested that studying responses to fearful stimuli might yield insights into the relation between (prenatal exposure to) state and trait anxiety. Bishop et al. (2004) found that individuals with high state anxiety respond stronger to fearful stimuli. Based on our P350 and - albeit to a lesser extent - P150 findings, one may suggest that not only individuals with high(er) levels of state anxiety, but also individuals with high(er) levels of general anxiety may respond stronger to fearful stimuli. In addition, combining the association we found between exposure to general anxiety and alterations in multimodal emotion processing, with the finding that high trait anxiety has also been linked to altered audio-visual processing (Koizumi et al., 2011), one might deduce that general anxiety and trait anxiety have (some) similar characteristics and/or related underlying mechanisms.

In the current study, no evidence was found for an association between prenatal exposure to maternal anxiety and alterations in the processing of multimodal emotional information. One possible explanation is that the present paradigm was not sensitive enough to measure the association. In evidence of this, only for the P150, instead of for all areas under investigation, a (small) interaction between Visual Prime and Auditory Emotion was found (see Table 2), indicating integration of the emotional information from face and voice. In other words, maybe no multimodal processing had taken place, precluding conclusions on how prenatal exposure to maternal anxiety may affect the processes involved. Future studies could develop a more sensitive paradigm, so that firmer conclusions may be drawn about the possible association between prenatal exposure to anxiety and processing of emotional information from face and voice.

A drawback of the study is that the effects we found were not very large; The partial $\eta^{2}$ values ranged between .034 and .050 . However, results remained intact after controlling for a number of potential confounders, including postpartum anxiety levels. In addition, we investigated a rather large group of infants $(N=82)$, who attended the visual stimuli quite well (73.2\% on average), and we were able to retain a relatively large average number of stimuli per condition (37.3). This suggests that although the effects we found may be small, they appear to be robust.

In summary, the current study investigated multimodal processing of fearful and happy face/voice pairs in infants in the context of prenatal exposure to maternal anxiety. Analysis revealed higher P350 and, to a lesser extent, P150 amplitudes in response to fearful vocalisations when infants had been exposed to higher levels of anxiety, irrespective of the emotion in the visual prime. This suggest that these infants may allocate more attention to fearful stimuli and that they may be aroused more easily than infants exposed to lower levels of anxiety These findings are compatible with the hypothesis that prenatal exposure to maternal anxiety may lead to more extensive processing of fear-related stimuli.

\section{Acknowledgments}

The authors are grateful to the parents and infants for their continued participation in our longitudinal study. In addition, they thank Dr. Tobias Grossmann for his advice on the multimodal paradigm, and Prof. Dr. I. Winkler for reading and commenting on previous drafts of the paper. Also, the authors are obliged to Marion van den Heuvel, Malou Menting, and Jaimy Sengers for their help in scoring the videos, and to the students who helped with data collection and processing.

The PELS study is supported by the national funding agencies of the European Science Foundation participating in the Eurocores Program EuroSTRESS programme, i.e. the Brain and Cognition Programme of the Netherlands Organisation for Scientific Research (NWO) for the Netherlands. The PELS study was initiated by BVdB and conducted in collaboration with Vivette Glover (Imperial College London), Stephan Claes (KU Leuven) and Alina Rodriguez (Uppsala University Sweden). BVdB is supported by European Commission Seventh Framework Programme (FP7HEALTH.2011.2.2.2-2 BRAINAGE, Grant agreement no: 279281).

\section{Appendix A. Supplementary material}

Supplementary data associated with this article can be found, in the online version, at http://dx.doi.org/10.1016/j.bandc.2014. 12.001 .

\section{References}

Altman, D. G., \& Royston, P. (2006). The cost of dichotomising continuous variables. BMJ, 332, 1080 .

Anderson, A. L., Marois, R., Colson, E. R., Peterson, B. S., Duncan, C. C., Ehrenkranz, R. A., et al. (2001). Neonatal auditory activation detected by functional magnetic resonance imaging. Magnetic Resonance Imaging, 19(1), 1-5.

Anderson, A. L., \& Thomason, M. E. (2013). Functional plasticity before the cradle: A review of neural functional imaging in the human fetus. Neuroscience and Biobehavioral Reviews, 37(2220-2232). 
Arrindell, W. A., \& Ettema, J. H. M. (2003). Symptom checklist SCL-90: Handleiding bij een multidimensionele psychopathologie-indicator [Symptom Checklist SCL-90: Manual to a multidimensional psychopathology-indicator]. Netherlands: Swets Test Publishers.

Bar-Haim, Y. (2010). Research review: Attention bias modification (ABM): A novel treatment for anxiety disorders. Journal of Child Psychology and Psychiatry, 51, 859-870.

Beard, J. L. (2008). Why iron deficiency is important in infant development. The Journal of Nutrition, 138, 2534-2536.

Bergman, K., Sarkar, P., O'Connor, T. G., Modi, N., \& Glover, V. (2007). Maternal stress during pregnancy predicts cognitive ability and fearfulness in infancy. Journal of the American Academy of Child and Adolescent Psychiatry, 46, 1454-1463.

Bishop, S. J., Duncan, J., \& Lawrence, A. D. (2004). State anxiety modulation of the amygdala response to unattended threat-related stimuli. The Journal of Neuroscience, 24, 10364-10368.

Čeponienè, R., Lepistö, T., Shestakova, A., Vanhala, R., Alku, P., Näätänen, R., et al. (2003). Speech-sound-selective auditory impairment in children with autism: They can perceive but do not attend. Proceedings of the National Academy of Sciences, 100, 5567-5572.

Charil, A., LaPlante, D. P., Vaillancourt, C., \& King, S. (2010). Prenatal stress and brain development. Brain Research Reviews, 65, 56-79.

Davis, E. P., \& Sandman, C. A. (2010). The timing of prenatal exposure to maternal cortisol and psychosocial stress is associated with human infant cognitive development. Child Development, 81, 131-148.

de Gelder, B., Pourtois, G., \& Weiskrantz, L. (2002). Fear recognition in the voice is modulated by unconsciously recognized facial expressions but not by unconsciously recognized affective pictures. Proceedings of the National Academy of Sciences, 99, 4121-4126.

de Gelder, B., \& Vroomen, J. (2000). The perception of emotions by ear and by eye. Cognition and Emotion, 14, 289-311.

De Kloet, E. R., Joëls, M., \& Holsboer, F. (2005). Stress and the brain: From adaptation to disease. Nature Reviews Neuroscience, 6, 463-475.

DeBoer, T., Scott, L., \& Nelson, C. (2007). Methods for acquiring and analyzing infant event-related potentials. In M. de Haan (Ed.), Infant EEG and event-related potentials (pp. 5-37). New York: Psychology Press.

Derogatis, L. R., \& Cleary, P. A. (1977). Confirmation of the dimensional structure of the SCL-90: A study in construct validation. Journal of Clinical Psychology, 33, 981-989.

Derogatis, L. R., Lipman, R. S., \& Covi, L. (1973). SCL-90: An outpatient psychiatric scale: Preliminary report. Psychopharmacology Bulletin, 9, 13-27.

Entringer, S., Buss, C., \& Wadhwa, P. D. (2010). Prenatal stress and developmental programming of human health and disease risk: Concepts and integration of empirical findings. Current Opinion in Endocrinology, Diabetes, and Obesity, 17, 507-516.

Erwin, R., \& Buchwald, J. (1986). Mid-latency auditory evoked responses: Differential effects of sleep in the human. Electroencephalography and Clinical Neurophysiology/Evoked Potentials Section, 65, 383-392.

Fellman, V., Kushnerenko, E., Mikkola, K., Čeponienè, R., Leipälä, J., \& Näätänen, R. (2004). Atypical auditory event-related potentials in preterm infants during the first year of life: A possible sign of cognitive dysfunction. Pediatric Research, 56, 291-297.

Flom, R., \& Bahrick, L. (2007). The development of infant discrimination in multimodal and unimodal stimulation: The role of inter-sensory redundancy. Developmental Psychology, 43, 238-252.

Georgieff, M. K. (2008). The role of iron in neurodevelopment: Fetal iron deficiency and the developing hippocampus. Biochemical Society Transactions, 36, $1267-1271$.

Georgieff, M. K., Wewerka, S. W., Nelson, C. A., \& deRegnier, R.-A. (2002). Iron status at 9 months of infants with low iron stores at birth. The Journal of Pediatrics, 141, 405-409.

Gillies, G. E., Virdee, K., McArthur, S., \& Dalley, J. W. (2014). Sex-dependent diversity in ventral tegmental dopaminergic neurons and developmental programming: A molecular, cellular and behavioural analysis. Neuroscience, 282, 69-85.

Gluckman, P. D., Hanson, M. A., \& Buklijas, T. (2010). A conceptual framework for the developmental origins of health and disease. Journal of Developmental Origins of Health and Disease, 1(01), 6-18.

Graignic-Philippe, R., Dayan, J., Chokron, S., Jacquet, A. Y., \& Tordjman, S. (2014). Effects of prenatal stress on fetal and child development: A critical literature review. Neuroscience and Biobehavioral Reviews, 43, 137-162.

Grossmann, T. (2010). The development of emotion perception in face and voice during infancy. Restorative Neurology and Neuroscience, 28, 219-236.

Grossmann, T., Striano, T., \& Friederici, A. D. (2006). Crossmodal integration of emotional information from face and voice in the infant brain. Developmental Science, 9, 309-315.

Harris, A., \& Seckl, J. (2011). Glucocorticoids, prenatal stress and the programming of disease. Hormones and Behavior, 59, 279-289.

Heron, J., O'Connor, T. G., Evans, J., Golding, J., \& Glover, V. (2004). The course of anxiety and depression through pregnancy and the postpartum in a community sample. Journal of Affective Disorders, 80, 65-73.

Hompes, T., Izzi, B., Gellens, E., Morreels, M., Fieuws, S., Pexsters, A., et al. (2013). Investigating the influence of maternal cortisol and emotional state during pregnancy on the DNA methylation status of the glucocorticoid receptor gene (NR3C1) promoter region in cord blood. Journal of Psychiatric Research, 47, $880-891$.

Kim, M. J., Loucks, R. A., Palmer, A. L., Brown, A. C., Solomon, K. M., Marchante, A. N., et al. (2011). The structural and functional connectivity of the amygdala: From normal emotion to pathological anxiety. Behavioural Brain Research, 223, 403-410.

Kodituwakku, P. (2007). Defining the behavioral phenotype in children with fetal alcohol spectrum disorders: A review. Neuroscience and Biobehavioral Reviews, 31, 192-201.

Koizumi, A., Tanaka, A., Imai, H., Hiramatsu, S., Hiramoto, E., Sato, T., et al. (2011). The effects of anxiety on the interpretation of emotion in the face-voice pairs. Experimental Brain Research, 213, 275-282.

Kotsoni, E., de Haan, M., \& Johnson, M. H. (2001). Categorical perception of facial expressions by 7-month-old infants. Perception, 30, 1115-1126.

Kushnerenko, E., Ceponienè, R., Balan, P., Fellman, V., Huotilainen, M., \& Näätänen, R. (2002). Maturation of the auditory event-related potentials during the first year of life. NeuroReport, 13, 47-51.

Lavoie, M. E., Robaey, P., Stauder, J. E., Glorieux, J., \& Lefebvre, F. (1998). Extreme prematurity in healthy 5-year-old children: A re-analysis of sex effects on event-related brain activity. Psychophysiology, 35, 679-689.

Leppänen, J. M., \& Nelson, C. A. (2012). Early development of fear processing. Current Directions in Psychological Science, 21, 200-204.

Luck, S. J. (2005). An introduction to the event-related potential technique. Cambridge, MA: The MIT Press.

Lupien, S. J., McEwen, B. S., Gunnar, M. R., \& Heim, C. (2009). Effects of stress throughout the lifespan on the brain, behaviour and cognition. Nature Reviews Neuroscience, 10, 434-445.

MacCallum, R. C., Zhang, S., Preacher, K. J., \& Rucker, D. D. (2002). On the practice of dichotomization of quantitative variables. Psychological Methods, 7, 19-40.

Malaspina, D., Corcoran, C., Kleinhaus, K., Perrin, M., Fennig, S., Nahon, D., et al. (2008). Acute maternal stress in pregnancy and schizophrenia in offspring: A cohort prospective study. BMC Psychiatry, 8.

Mennes, M., Stiers, P., Lagae, L., \& Van den Bergh, B. (2006). Long-term cognitive sequelae of antenatal maternal anxiety: Involvement of the orbitofrontal cortex. Neuroscience and Biobehavioral Reviews, 30, 1078-1086.

Mennes, M., Van den Bergh, B., Lagae, L., \& Stiers, P. (2009). Developmental brain alterations in 17-year-old boys are related to antenatal maternal anxiety. Clinical Neurophysiology, 120, 1116-1122.

Monk, C., Fitelson, E. M., \& Werner, E. (2011). Mood disorders and their pharmacological treatment during pregnancy: Is the future child affected? Pediatric Research, 69, 3R-10R.

Montague, D. P., \& Walker-Andrews, A. S. (2001). Peekaboo: A new look at infants perception of emotion expressions. Developmental Psychology, 37, 826.

Nelson, C. A., \& Bloom, F. E. (1997). Child development and neuroscience. Child Development, 68, 970-987.

Nelson, C. A., Wewerka, S., Thomas, K. M., deRegnier, R. A., Tribbey-Walbridge, S., \& Georgieff, M. (2000). Neurocognitive sequelae of infants of diabetic mothers. Behavioral Neuroscience, 114, 950.

O'Connor, T. G., Heron, J., Golding, J., Beveridge, M., \& Glover, V. (2002). Maternal antenatal anxiety and children's behavioural/emotional problems at 4 years. Report from the Avon longitudinal study of parents and children. The British Journal of Psychiatry, 180, 502-508.

O’Donnell, K. J., Bugge Jensen, A., Freeman, L., Khalife, N., O’Connor, T. G., \& Glover, V. (2012). Maternal prenatal anxiety and downregulation of placental11 $\beta$-hsd2. Psychoneuroendocrinology, 37, 818-826.

Oitzl, M., Champagne, D., van der Veen, R., \& de Kloet, E. R. (2010). Brain development under stress: Hypotheses of glucocorticoid actions revisited Neuroscience and Biobehavioral Reviews, 34, 853-866.

Otte, R. A., Braeken, M. A. K. A., Winkler, I., \& Van den Bergh, B. R. H. (2011). Effects of maternal anxiety during pregnancy on novelty processing in 2-month-old infants. An ERP study. Journal of Developmental Origins of Health and Disease, 2(supplement S1), S35-S150.

Otte, R. A., Donkers, F. C., Braeken, M. A. K. A., \& Van den Bergh, B. R. H. (2015). Multimodal processing of emotional information in 9-month-old infants II: Prenatal exposure to maternal anxiety. Brain and Cognition, 95, 107-117.

Otte, R. A., Winkler, I., Braeken, M. A. K. A., \& Van den Bergh, B. R. H. (submitted for publication). Prenatal early life stress and auditory information processing in 9month-olds.

Otte, R. A., Winkler, I., Braeken, M. A. K. A., Stekelenburg, J. J., Stelt, O., \& Van den Bergh, B. R. H. (2013). Detecting violations of temporal regularities in waking and sleeping two-month-old infants. Biological Psychology, 92, 315-322.

Peltola, M. J., Leppänen, J. M., Mäki, S., \& Hietanen, J. K. (2009). Emergence of enhanced attention to fearful faces between 5 and 7 months of age. Social Cognitive and Affective Neuroscience, 4, 134-142.

Ploeg, H. v. d, Defares, P., \& Spielberger, C. (1980). Handleiding bij de ZelfBeoordelingsvragenlijst ZBV: Een Nederlandstalige bewerking van de Spielberger State-TraitAnxiety Inventory. [Manual of the Self-Evaluation Questionnaire: A Dutch version of the State-Trait Anxiety Inventory]. Swets \& Zeitlinger B.V.: Lisse, The Netherlands.

Polich, J. (2007). Updating P300: An integrative theory of P3a and P3b. Clinical Neurophysiology, 118, 2128-2148.

Purhonen, M., Kilpeläinen-Lees, R., Valkonen-Korhonen, M., Karhu, J., \& Lehtonen, J. (2004). Cerebral processing of mother's voice compared to unfamiliar voice in 4-month-old infants. International Journal of Psychophysiology, 52, 257-266.

Räikkönen, K., Seckl, J. R., Pesonen, A.-K., Simons, A., \& Van den Bergh, B. R. H. (2011) Stress, glucocorticoids and liquorice in human pregnancy: Programmers of the offspring brain. Stress, 14, 590-603.

Ramsey, J. L., Langlois, J. H., \& Marti, N. C. (2005). Infant categorization of faces: Ladies first. Developmental Review, 25, 212-246. 
Rice, F., Harold, G., Boivin, J., Van den Bree, M., Hay, D., \& Thapar, A. (2010). The links between prenatal stress and offspring development and psychopathology: Disentangling environmental and inherited influences. PsychologicalMedicine, $40,335-345$.

Rodriguez, A., \& Bohlin, G. (2005). Are maternal smoking and stress during pregnancy related to ADHD symptoms in children? Journal of Child Psychology and Psychiatry, 46, 246-254.

Rosen, J. B., \& Schulkin, J. (1998). From normal fear to pathological anxiety. Psychological Review, 105, 325.

Royston, P., Altman, D. G., \& Sauerbrei, W. (2006). Dichotomizing continuous predictors in multiple regression: A bad idea. Statistics in Medicine $25,127-141$

Sandman, C. A., Davis, E. P., Buss, C., \& Glynn, L. M. (2011). Prenatal programming of human neurological function. International Journal of Peptides, 2011, 9.

Siddappa, A. M., Georgieff, M. K., Wewerka, S., Worwa, C., Nelson, C. A., \& deRegnier, R.-A. (2004). Iron deficiency alters auditory recognition memory in newborn infants of diabetic mothers. Pediatric Research, 55, 1034-1041.

Spielberger, C., Gorsuch, R., \& Lushene, R. (1970). STAI manual for the state-trait anxiety inventory. Palo Alto, CA: Consulting Psychologists Press.

Spielberger, C. (1975). The measurement of state and trait anxiety: Conceptual and methodological issues. In L. Levi (Ed.), Emotions: Their parameters and measurement (pp. 713-725). New York: Raven.

Streissguth, A. P., Barr, H. M., \& Martin, D. C. (1983). Maternal alcohol use and neonatal habituation assessed with the Brazelton scale. Child Development, 54, 1109-1118.

Sussman, E. (2007). A new view on the MMN and attention debate: Auditory context effects. Journal of Psychophysiology, 21, 164-175.

Van den Bergh, B. R. H. (2011). Developmental programming of early brain and behaviour development and mental health: A conceptual framework. Developmental Medicine and Child Neurology, 53, 19-23.

Van den Bergh, B. R. H., \& Marcoen, A. (2004). High antenatal maternal anxiety is related to ADHD symptoms, externalizing problems, and anxiety in 8-and 9year-olds. Child Development, 75, 1085-1097.

Van den Bergh, B. R. H., Mennes, M., Stevens, V., Meere, J. Van der, Borger, N., Stiers P., et al. (2006). ADHD deficit as measured in adolescent boys with a continuous performance task is related to antenatal maternal anxiety. Pediatric Research, 59, 78-82.
Van den Bergh, B. R. H., Mulder, E. J. H., Mennes, M., \& Glover, V. (2005). Antenatal maternal anxiety and stress and the neurobehavioural development of the fetus and child: Links and possible mechanisms. A review. Neuroscience $\mathcal{\sigma}^{\prime}$ Biobehavioral Reviews, 29, 237-258.

Van den Bergh, B. R. H., Otte, R. A., Braeken, M. A. K. A., van den Heuvel, M. I., \& Winkler, I. (2012). Prenatal exposure to maternal anxiety is associated with sensory-cognitive development in 2-month-olds. Developmental Medicine and Child Neurology, 54(Supplement 2), 22.

Van den Bergh, B. R. H., Otte, R. A., Braeken, M. A. K. A., van den Heuvel, M. I., \& Winkler, I. (2013). Does prenatal exposure to maternal anxiety influence information processing in two-month-old infants? An auditory ERP study. Biological Psychiatry, 73(9), 132.

van den Heuvel, M. I., Donkers, F. C. L., Winkler, I., Otte, R. A., \& Van den Bergh, B. R. H. (2014). Maternal mindfulness and anxiety during pregnancy affect infants' neural responses to sounds. Social Cognitive E Affective Neuroscience. http:// dx.doi.org/10.1093/scan/nsu075.

van den Heuvel, M. I., Otte, R. A., Braeken, M. A. K. A., Winkler, I., Kushnerenko, E., \& Van den Bergh, B. R. H. (submitted for publication). Detecting violations of temporal regularities and novelty in waking two and four month old infants: An auditory ERP study.

Van den Stock, J., Grèze, J., \& de Gelder, B. (2008). Human and animal sounds influence recognition of body language. Brain Research, 1242, 185-190.

Vogel, M., Monesson, A., \& Scott, L. S. (2012). Building biases in infancy: The influence of race on face and voice emotion matching. Developmental Science, 15, 359-372.

Walker-Andrews, A. S., \& Lennon, E. (1991). Infants' discrimination of vocal expressions: Contributions of auditory and visual information. Infant Behavior and Development, 14, 131-142.

Weikum, W. M., Oberlander, T. F., Hensch, T. K., \& Werker, J. F. (2012). Prenatal exposure to antidepressants and depressed maternal mood alter trajectory of infant speech perception. Proceedings of the National Academy of Sciences, 109, $17221-17227$.

Weinstock, M. (2008). The long-term behavioural consequences of prenatal stress Neuroscience and Biobehavioral Reviews, 32, 1073-1086.

Wyrwoll, C. S., Holmes, M. C., \& Seckl, J. R. (2011). 11ß-hydroxysteroidde hydrogenases and the brain: From zero to hero, a decade of progress. Frontiers in neuroendocrinology, 32, 265-286. 